\title{
A NOVEL RADIATION ENHANCED TRAVELING WAVE FEED CIRCULAR POLARIZED ANTENNA
}

\author{
Anne D. Abeygunasekera ${ }^{* 1}$, Charles E. Free ${ }^{1}$, Mikko Komulainen ${ }^{2}$ \\ ${ }^{1}$ Advanced Technology Institute, University of Surrey, Guildford, Surrey, GU2 \\ 7XH, United Kingdom \\ ${ }^{2}$ Microelectronics and Materials Physics Laboratories, Dept of Electronic \\ Engineering, University of Oulu, Linnanmaa PO BOX 4500 90014, Finland
}

\section{Introduction}

The expanding applications for microwave circuits in current communication systems has arisen the need for high density packaging. Circuit technologies often need the antenna to be of small scale whilst offering high performance. In this paper, we propose a novel antenna structure capable of high radiation efficiency suitable for use in multilayer microwave circuits. The traveling wave feed system offers high quality circular polarization which is a key feature of many existing and developing mobile communication systems. The proposed design can be fabricated using thick film technology, thus satisfying the high volume demand of microwave circuits at a lower cost.

The proposed antenna uses a low permittivity dielectric layer on a high permittivity substrate to improve the fringing fields at the edges of the radiation patches. The work is an extension of the concept using strips of low dielectric constant material underneath the radiation edges of a patch to enhance radiation efficiency[1]. In the proposed design the low dielectric constant material is placed above the high permittivity substrate forming a multilayer structure. The antenna design uses a circular slotline channel beneath the high permittivity substrate to excite the rectangular patches. A theoretical investigation based on electromagnetic simulation was performed to validate the new radiation efficiency enhanced antenna at $10 \mathrm{GHz}$.

\section{Antenna Structure And Theory}

The structure of the radiation enhanced circular polarized antenna is illustrated in figure[1]. The configuration of the antenna is composed of 8 planar rectangular radiating patches. Each patch is linearly polarized and consists of dimensions $L_{p}$ and $W_{p}$, where $L_{p}$ is the resonant dimension and $W_{p}$ is the width of the patch, the radiating edge. The patches are excited with a progressive phase lag of $45^{\circ}$ because there is an annular spacing of $\lambda / 8$ between the patches, thus leading to circular polarization. The basic design concept of the antenna was originally established by K. Lum and C. Free[2]. The antenna feed is by a circular slotline channel, of width $\mathrm{W}_{\mathrm{s}}$, runs in the ground plane beneath the patches. The slotline is excited through a conventional microstrip-to-slotline transition. The slotline is offset by an increment of $120 \mu \mathrm{m}$ so that a portion of the signal travelling in the slotline will successively excite each patch, therefore compensating for the progressive loss of signal in the slotline. 
The test antenna simulation was carried out on Alumina ADS-96R with the following parameters: Substrate thickness $\mathrm{h}=0.76 \mathrm{~mm}$, Substrate dielectric constant $\varepsilon_{\mathrm{r}}=9.6$. The key antenna dimensions:

$\begin{array}{ll}\text { Length of the patch } \quad W_{p} & =6.9 \mathrm{~mm} \\ \text { Width of the patch } \quad L_{p} & =2.9 \mathrm{~mm} \\ \text { Width of the slotline } \quad \mathrm{W}_{\mathrm{s}} & =0.4 \mathrm{~mm}\end{array}$

Diameter of the circular slotline channel $=37 \mathrm{~mm}$

Circuit dimensions $\quad=50.8 \mathrm{~mm} \times 50.8 \mathrm{~mm}$

As illustrated in figure[2] a low dielectric constant material was placed beneath the patches to improve the efficiency of the radiation. One of the key parameters of radiation loss is the dielectric constant. The lower the dielectric constant the less the concentration of energy is in the substrate region. Therefore more radiation loss occurs. The high dielectric constant substrate material reduces the radiation loss. This is because the most of the EM field is concentrated in the dielectric between the conductive patch and the ground plane. The low dielectric constant material used was KQ150(Heraeus Inc) with a dielectric constant of 3.9. Dielectric layer thickness was set to $50 \mu \mathrm{m}$. The presence of the low dielectric material increases the intensity of the electric field distribution at the radiating patch edges.

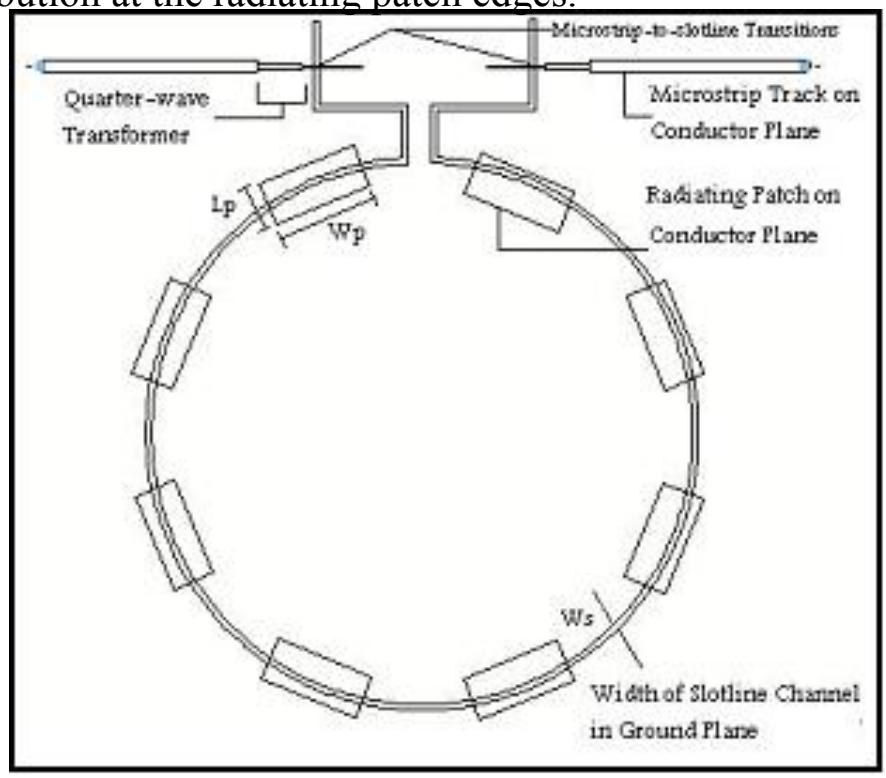

Figure[1] - Configuration of the antenna

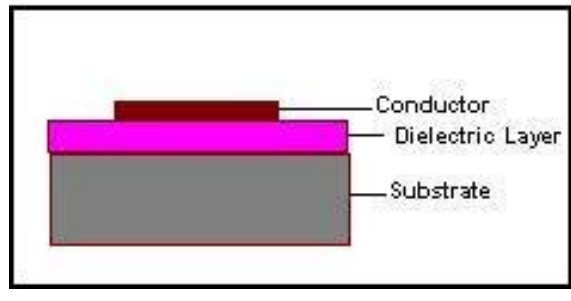

Figure[2] - Configuration of the layers

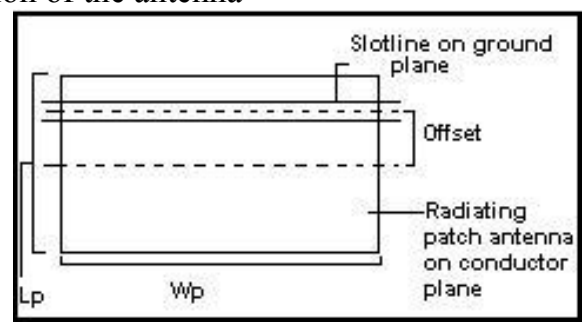

Figure[3]-Definition of Incremental Offset between slotline channel and patch 


\section{Simulation Results}

The simulation was carried out using AGILENT Advanced Design System (ADS) software which is a computer aided design (CAD) tool.

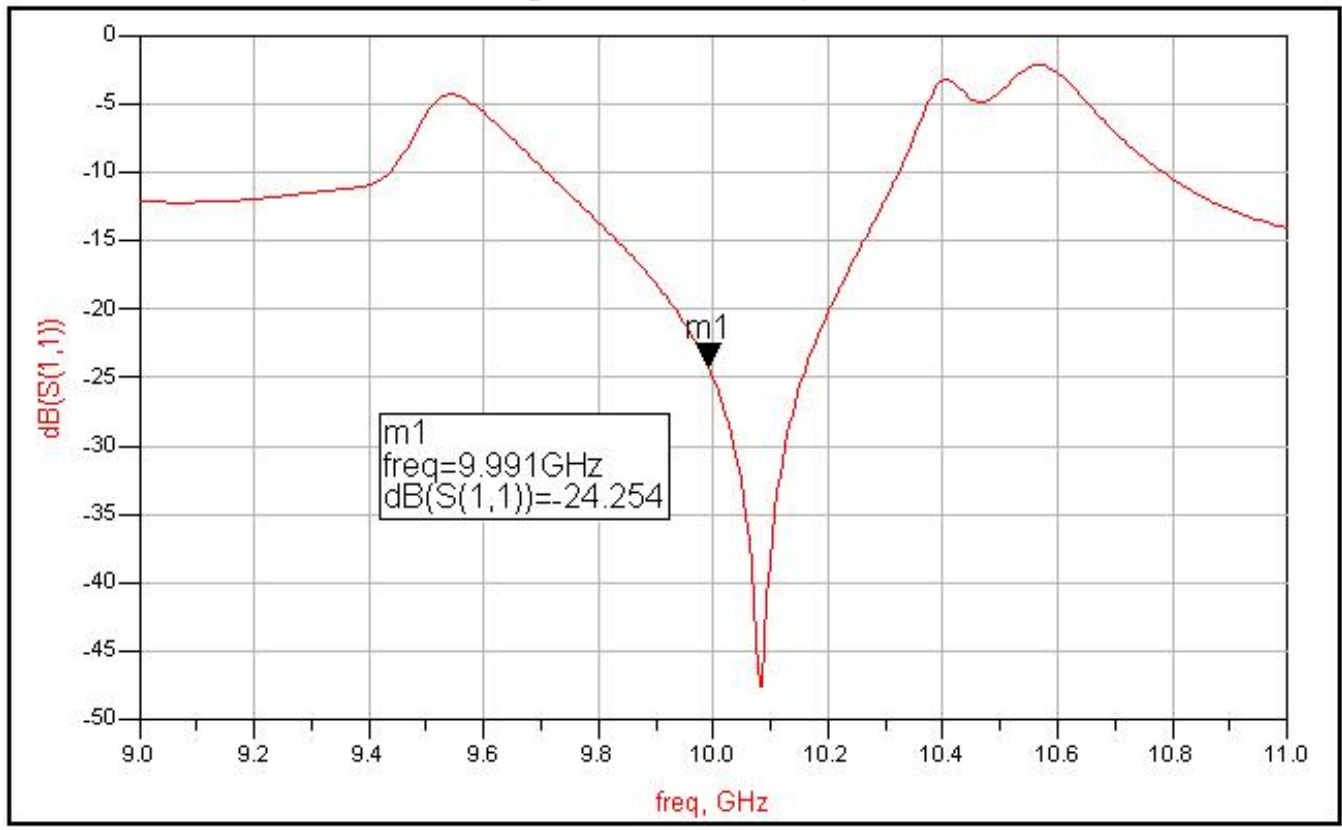

Figure[4]-Antenna without the dielectric layer-Return loss

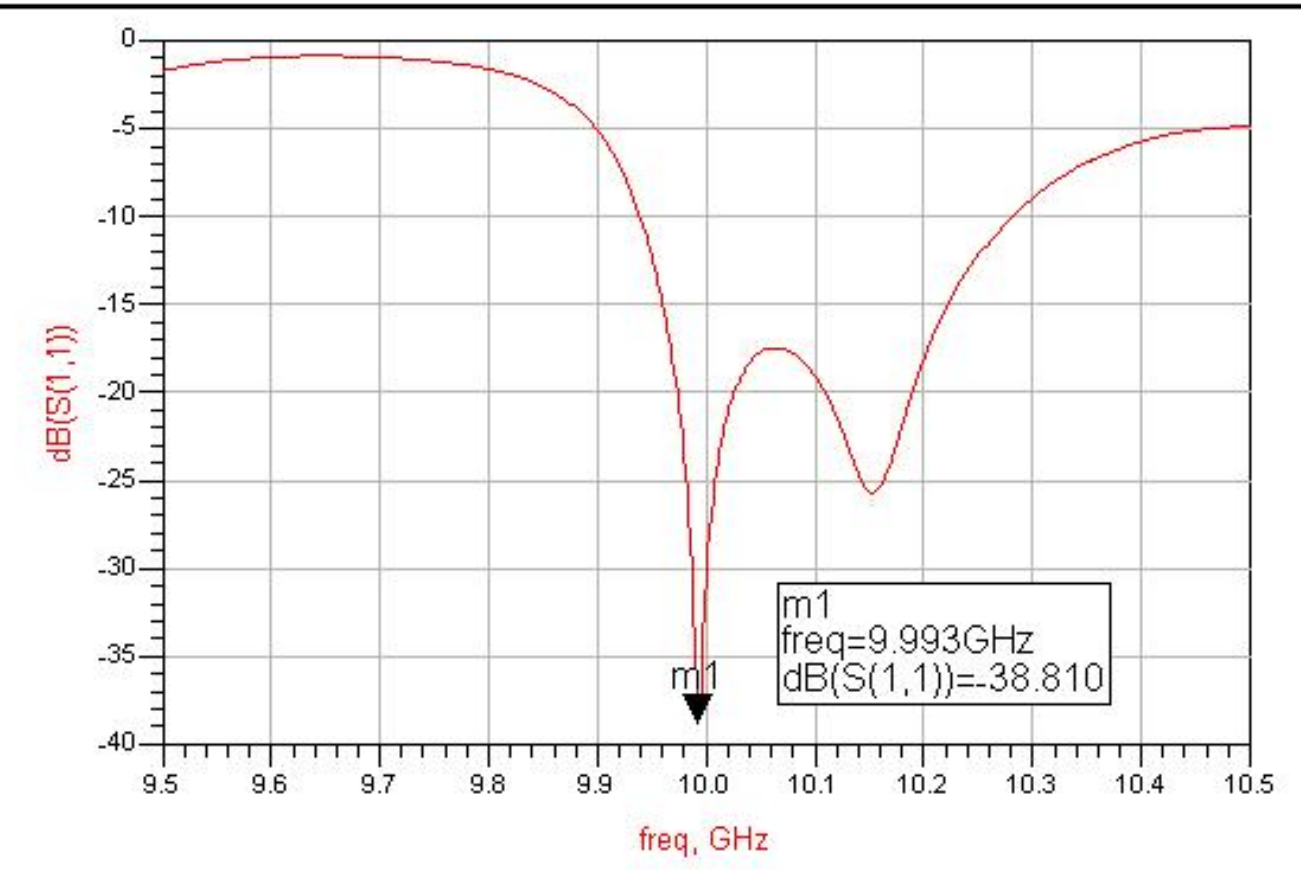

Figure[5]-Antenna with the Dielectric layer-Return loss

ANTENNA EFFICIENCY

\begin{tabular}{|l|c|}
\hline Antenna without the Dielectric & $74 \%$ \\
\hline Antenna with the Dielectric & $86 \%$ \\
\hline
\end{tabular}


Considering the $\mathrm{CP}$ antenna without the low dielectric constant layer, the optimum frequency of operation is at $10.08 \mathrm{GHz}$. This is slightly different from the designed value of $10 \mathrm{GHz}$ because no matching was included to account for the reactance presented by the patches to the feed line. As seen from figure[4], the return loss of the antenna is $-24.25 \mathrm{~dB}$ at $9.9 \mathrm{GHz}$. As seen from figure[5], the return loss of the antenna with the low dielectric constant material underneath its patches, is $-38.8 \mathrm{~dB}$ at the optimum frequency of operation which is $9.9 \mathrm{GHz}$. It can be observed that the return loss demonstrates an increment of $60 \%$ at $9.9 \mathrm{GHz}$. The radiation efficiency has increased by $12 \%$. It was found that an adequate combination of good return loss and radiation efficiency existed in the new multilayer antenna structure.

\section{Conclusion}

In this paper a radiation enhancement method using low dielectric constant material is demonstrated. Excellent return loss and improved radiation efficiency was obtained from the novel multilayer design structure. The proposed structure is suitable for compact multilayer microwave structures.

\section{References}

1. K.M. Lum; T. Tick; C. Free; H. Jantunen, Design and Measurement Data for a Microwave Dual-CP Antenna Using a New Travelling-Wave Feed Concept, IEEE Trans. On Microwave Theory and Techniques, Vol. 54, no. 6, pp. 2880-2886, June 2006

2. K. M. Lum, C. Laohapensaeng, and C. E. Free, "A novel travelingwave feed technique for circularly polarized planar antennas," IEEE Microwave Wireless Components Letters, vol. 15, no. 3, pp. 180-182, March 2005

3. K. P. Tang, C. E. Free, and G. F. Goldspink, "A novel slotline-fed microstrin patch antenna", IEEE Antennas and Propagation Conf:, vol. 1, no. 407, pp. 235-238, April 1995.

4. J. Huang, "A technique for an array to generate circular polarization with linearly polarized elements", IEEE Trans. Antennas and Propagation., vol. 34, no. 9, pp. 1113-1124, September 1986.

5. E. A. Mariani, C. P. Heinzman, J. P. Agrios, and S. B. Cohn, "Slot line characteristic", IEEE Trans. Microwave Theory Tech., vol. 17, no 12, pp. 1091-1096, December 1969.

6. J. B. Knorr, "Slot-line transitions", IEEE Trans. Microwave Theory Tech., vol. 22, no. 5, pp. 548-554, May 1974.

7. K. M. Lum, and C. E. Free, A Novel Traveling-Wave feed Technique for Circularly Polarized Planar Microstrip antennas, IEEE Antennas and Propagation Conf., vol. 2A, pp.250 - 253, July 2005 